\title{
Advanced Metal Lift-offs and Nanoimprint for Plasmonic Metal Patterns
}

\author{
Sangkeun Sung', Chul-Hyun Kim', Jihye Lee', Joo-Yun Jung', Jun-ho Jeong', \\ Jun-Hyuk Choi ${ }^{1, *}$, and Eung-Sug Lee ${ }^{1}$ \\ 1 Korea Institute of Machinery \& Materials, 156 Gajeongbuk-Ro, Yuseong-Gu, Daejeon 305-343, South Korea \\ \# Corresponding Author / E-mail: junhyuk@kimm.re.kr, TEL: +82-2-868-7817, FAX: +82-2-868-7123
}

KEYWORDS: Nanoimprint, Life-off process, Rabbit ears, Reverse-tapered profile, Transmittance

\begin{abstract}
An effective and reliable fabrication approach for silver nanostructure arrays was developed using UV nanoimprint with a lift-off resist and a Si-based etch mask for enhanced plasmonic resonance-driven device performance. Since a tapered nanopillar array is imprinted on the resist, by coating it with a Si-based etch mask layer and carrying out the etching procedure, a reverse-tapered nanohole array for metal deposition can be crated. Compared to the conventional lift-off process, a more tolerable window of lifeoff process conditions is possible, and dot patterns with high-aspect-ratio thickness profiles and no rabbit ears can be fabricated. Simulation results corresponding to dot diameters of 150,235, and $265 \mathrm{~nm}$ (twice those in their period) were qualitatively in good agreement with experimental results in terms of transmittance. This suggests that the proposed technique is a fairly reliable and accessible fabrication strategy for applications.
\end{abstract}

\section{Introduction}

Metallic nanostructure arrays have recently attracted growing interest because of their potential impact on various optoelectronic devices and sensors. Metallic nanostructures, often referred to as metallic photonic crystals, can serve as light-scattering centers through plasmonic resonant excitation and out-coupling by the incident electromagnetic field, to enhance the resonant plasmonic field and outcoupled emissions. Hence, they can contribute to increased energy transformation efficiency in optoelectronic devices, ${ }^{1-4}$ also enhancing the sensing quality ${ }^{5-7}$ via the increased field intensity and sensitivity. Additional applications include a wide range of metallic conductorbased electronic devices, including memory ${ }^{8,9}$ and optical modulation. ${ }^{10-12}$

Advances in nanofabrication technologies have led to a diversity of research accomplishments. Typical nanofabrication approaches are based on well-established microfabrication tools, such as lift-off ${ }^{13-15}$ and direct ion metal milling. ${ }^{16,17}$ However, to make these techniques work at the nanoscale level, either advanced optimization or a derived breakthrough technology must be developed, since existing process recipes can only be properly optimized at the microscale level. Metal lift-off outside the pattern trench can be facilitated if metal deposition at the pattern sidewall is restricted and the base pattern height increases at 1.5 times that of the imprint side. Hence, a persistent undercut profile pattern can be readily built up during microfabrication by tuning the dose intensity. However, this is hardly possible with the base nanopatterns achieved by nanoimprinting or other soft nanolithography techniques. ${ }^{18}$ To remedy this, an additional layer of lift-off resist (LOR) is often applied to the substrate prior to being coated with lithography resist. Since the LOR underlayer and lithography resist have different selective dissolution chemistries, the developing LOR can penetrate beneath the lithography resist pattern to form a naturally undercut profile favorable to metal lift-off. ${ }^{19-21}$ Similarly, nanoimprinting has been performed on a bilayer resist system composed of lift-off resist poly(methyl methacrylate) (LOR-PMMA), followed by removal of the imprint residue on the PMMA by either oxygen plasma etching ${ }^{19}$ or 
developer solution. ${ }^{20,21}$ Since LOR is dissolved isotropically, the developing LOR underlayer can be finely controlled beneath the imprinted pattern to prevent it from collapsing.

Unfortunately, this procedure demands restrictive wet-etching optimization, which offers no guarantee of stability or repeatability, due to its substantially sensitive nature. A reverse etching strategy, using a highly selective etching material such as silicon-based resist, was attempted. ${ }^{22-24}$ The imprinted pattern was typically formed with an overcut profile because of the facile mold release, making lift-off difficult. Hydrogen silsesquioxane (HSQ), a kind of spin-on glass (SOG), was applied ${ }^{22,23}$ as a coating to planarize the imprinted pattern holes, followed by etch-back of the HSQ to expose the top of the imprinted features. The high etching selectivity between HSQ and the imprint resist (more than a hundred) allowed dimensionally accurate pattern transfer down to the substrate via etch-down of the imprint resist through the HSQ layer. Finally, the overcut profile in the imprinted pattern was reversed to become an undercut profile suitable for a subsequent metal lift-off process. One of the early attempts to utilize spin-on glass for metal lift-off can be found, ${ }^{24}$ where SOG coated on polymer was directly imprinted, and then etched to expose the polymer underlayer. Due to the high etching selectivity, isotropic oxygen plasma etching of the exposed polymer was able to form an undercut polymer pattern with an undamaged SOG etch mask, facilitating metal lift-off. Nevertheless, these earlier approaches are dependent on individual empirical know-how, and thus are not stable enough or reliable enough for wider applications.

In the present work, a new process strategy for metal lift-off is presented, using a lift-off resist and a silicon-based resist for planarization and etch masking. Predefined patterns are formed by ultraviolet (UV) nanoimprinting on a UV-curable resist layer, followed by silicon resist planarization and etch-back. The presence of LOR beneath the UV resist facilitates the establishment of an undercut profile suitable for subsequent metal lift-off, allowing multiple choices for isotropic etch-down (either dry or wet). The optical properties of the processed metal patterns are characterized with respect to several different dot sizes to indirectly characterize their structural integrity.

\section{Experiments}

The overall process scheme is illustrated in Fig. 1. In the first stage, shown in Fig. 1(a), the soluble lift-off resist (LOR 1A, MicroChem, Inc.) and spin-coatable UV-curable nanoimprinting resist (LV300, Chemoptics, Inc.) were deposited in sequence on oxygen plasmatreated glass. Each layer was subjected to thermal annealing and solvent drying after spin-coating, and the layer thicknesses were optimized down to $100 \mathrm{~nm}$ for the LOR and $300 \mathrm{~nm}$ for the LV300. For the nanoimprinting, silicon master patterns were prepared to replicate the polymer mold on a glass backplate. To investigate the effects of hole size on metal optics and plasmonic absorption, we used three individual master pattern designs (stored in our laboratory), each consisting of a half-pitch hole array on a $100 \times 100 \mathrm{~mm}^{2}$ active pattern area of an 8 " silicon wafer, with respective hole diameters of 150, 235, and $265 \mathrm{~nm}$. The silicon master pattern was fabricated by $\mathrm{KrF}$ optical lithography, followed by a well-established anisotropic etching procedure, at the National Nanofab Center in Daejeon, Korea. The master patterns were replicated twice in turn to produce the same hole pattern array on a polyurethane acrylate-based custom-made UVcurable resin on a polyethylene terephthalate (PET) film, which was used as the nanoimprinting stamp. The mold resin consisted of tripropylene glycol diacrylate (Aldrich) and tri-methylol-propane triacrylate (Aldrich) as a monomer, with $4 \mathrm{wt} \%$ 2,2-dimethoxy-2phenylacetophenone (Aldrich) as the photoinitiator and 1-4 wt\% Rad 2200 N (TEGO Chemi Service) as the releasing agent.

As Fig. 1(b) shows, pillar-shaped pattern arrays were created by the UV nanoimprinting, which was carried out at a pressure of 0.2 bar under ultraviolet exposure (UV intensity of $13.6 \mathrm{~mW} / \mathrm{cm}^{2}$ ) for 90 seconds. Highly etch-selective silicon resist was spin-coated to have a thickness of $300 \mathrm{~nm}$, followed by annealing at $150^{\circ} \mathrm{C}$ for three minutes) over the imprinted pattern to fill and planarize the trench region, as shown in Fig. 1(c). In the next step, etch-back of the silicon resist was performed to open the imprinted pattern, using inductive coupled plasma (ICP) - reactive ion etching (RIE) with a gas mixture of $\mathrm{CHF}_{3}$ and $\mathrm{O}_{2}$ in a Multiplex ICP STS (Oxford Systems). Once the imprinted pattern was opened, as shown in Fig. 1(d), the etching conditions were changed to oxygen only at a reduced ICP power of $100 \mathrm{~W}$, to etch the imprint residue and LOR down to the substrate through the silicon resist etch mask. Hole pattern arrays were then generated, as shown in Fig. 1(e), for the silver deposition step shown in Fig. 1(f). The E-beam evaporation method (KVET-C500200, Korea Vacuum, Inc.) was used to apply a 30-nm coating of silver on top of $5 \mathrm{~nm}$ of chrome (to promote adhesion). Once again, the procedure was carried out at the National Nanofab Center in Korea. The silver layer, which was minimally deposited on the sidewall and top surface of the etch-down pattern, was removed by lift-off while being gently shaken in the developer $400 \mathrm{~K}$ (MicroChem, Inc.). The silver dot array was finally completed as shown in Fig. 1(g). The processed metal dot profiles were imaged via scanning electron microscopy (SEM) and atomic force microscopy (AFM), and were characterized in terms of the transmittance spectra for the three dot sizes, measured in the wavelength range of 300$1,000 \mathrm{~nm}$ in a NEOSYS-2000 (SCINCO,Inc.).

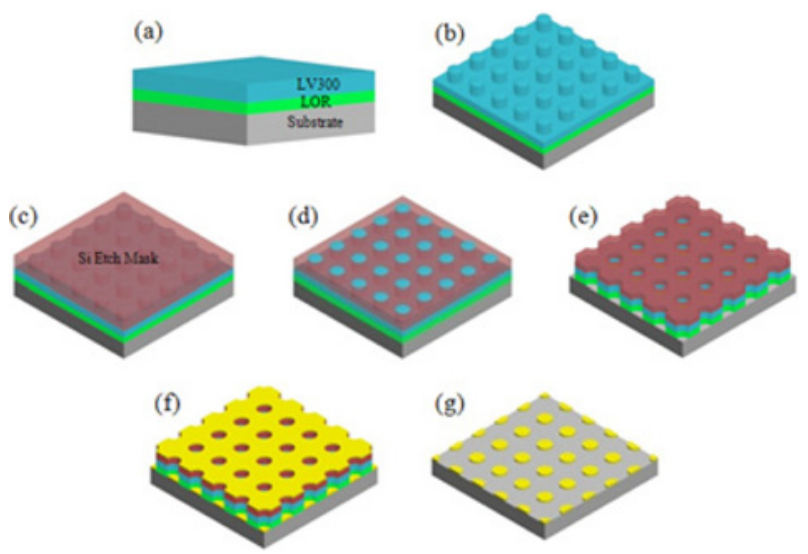

Fig. 1 Process scheme for metal pattern fabrication, utilizing a bilayer resist composed of a lift-off resist and a UV-curable resist for nanoimprinting, and a silicon-based resist for planarization and etch masking: a) coating of the lift-off resist and UV-curable resist, b) UV nanoimprinting, c) Si-based etch mask coating, d) etching of the etch mask, e) etching of the polymer resist through the etch mask, f) Ag deposition, and g) lift-off 


\section{Results and Discussion}

Images of the fabricated samples at several steps of the process are shown in Fig. 2. Fig. 1(b) shows nanopillar-structured arrays on the UV-nanoimprinting resist coated on top of the LOR. In Fig. 2(a), a distinct layer interface is observed, with neither mixing nor disruption, owing to the fact that the lift-off resist was sufficiently annealed before the UV-nanoimprint lithography (NIL) resist was applied. The thicknesses of the imprint residue and LOR were in the range of $100 \mathrm{~nm}$. As Fig. 2(b) shows, the nanoimprinted structures were planarized with Si-based etch mask material, followed by etch-back of the Si-based resist to barely expose the surface of the imprinted pillar pattern array (213 nm in diameter), corresponding to Fig. 1(d). Oxygen RIE was then used to remove the imprinted pattern and lift-off resist through the opened Si-based etch mask, and expose the substrate surface, as shown in Fig. 2(c, d), corresponding to Fig. 1(e). At this point, the hole size became larger at the bottom $(300 \mathrm{~nm})$ than at the top $(245 \mathrm{~nm})$. This reverse-tapered hole shape helps to prevent metal deposition on the inner sidewalls of the etched holes, so that metal lift off should ultimately be facilitated, although the dimensional accuracy of master pattern transfer may be sacrificed.

The completed silver nanopattern arrays after metal lift-off are shown in Fig. 3 for three different hole-type master patterns: $150 \mathrm{~nm}$ dot diameter, hexagonal array; $235 \mathrm{~nm}$ dot diameter, square array; and $265 \mathrm{~nm}$ dot diameter, hexagonal array. In comparison with the designed master pattern dimensions, the derived silver nanodot size was
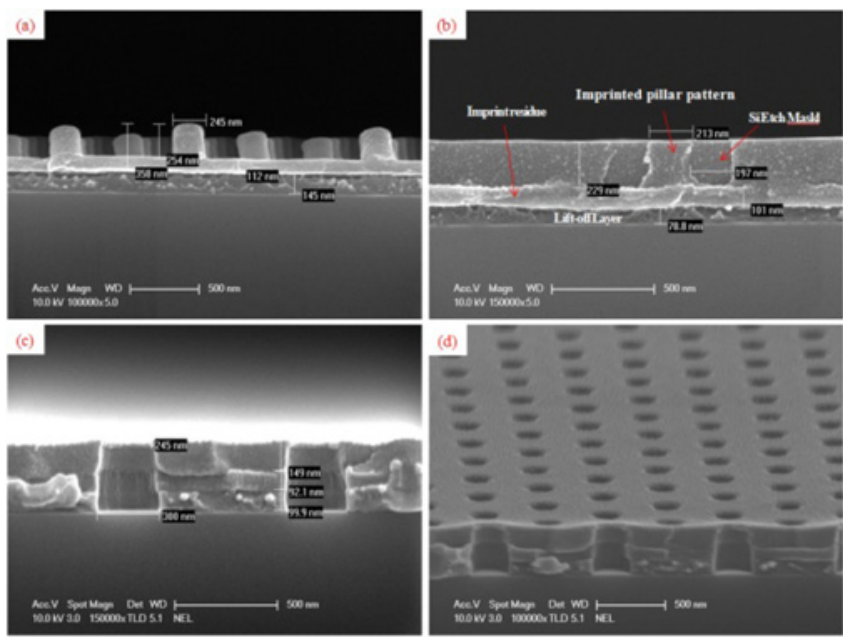

Fig. 2 SEM images of (a) the UV-imprinted pattern [Fig. 1(b)], (b) planarization using the silicon-based resist, followed by etch-back [Fig. 1(d)], (c) and (d) etch-down to the substrate via oxygen plasma etching [Fig. 1(e)]
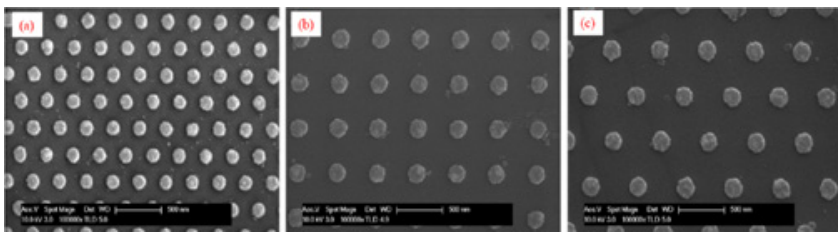

Fig. 3 Finalized metal dots (or patterns) of Fig. 1(g): (a) half-pitch, $150 \mathrm{~nm}$ dot diameter, (b) half-pitch, $235 \mathrm{~nm}$ dot diameter, and (c) halfpitch, $265 \mathrm{~nm}$ dot diameter diminished by $5 \%$ for $150 \mathrm{~nm}, 22 \%$ for $235 \mathrm{~nm}$, and $27 \%$ for $265 \mathrm{~nm}$. It is reasonably obvious that the dimensional deviations occurred during etch-down, as described above. A few scattered defects were observed, primarily in the $150 \mathrm{~nm}$ hexagonal array of Fig. 3(a), due to fabrication errors resulting from incomplete resist uniformity or lift-off. These fabrication deviations may be regarded as within the tolerable range (to be discussed in connection with the corresponding simulation results of (Fig. 5), but could be minimized via further process optimization. The sidewall configuration for the $265 \mathrm{~nm}$ hexagonal array master pattern, assessed by both AFM and SEM, exhibited no obvious rabbit ears, but only rounded dot shapes with actual thicknesses of 40-50 nm. It is believed that metal lift-off using neither lift-off resist nor Si based etch-mask resist would not achieve the nanodot thicknesses demanded in practical applications.

Fig. 5(a) shows transmittance measurement results for the metal nanodots of Fig 3 with respect to the atmosphere in the UV-visible
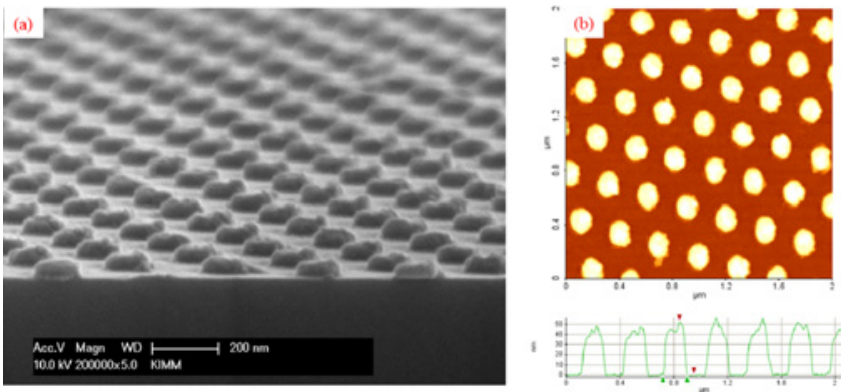

Fig. 4 Measurement of the hexagonally arrayed metal dot configuration with a dot diameter of $150 \mathrm{~nm}$, shown in Fig. 3(a): (a) tilted SEM images and (b) AFM profile
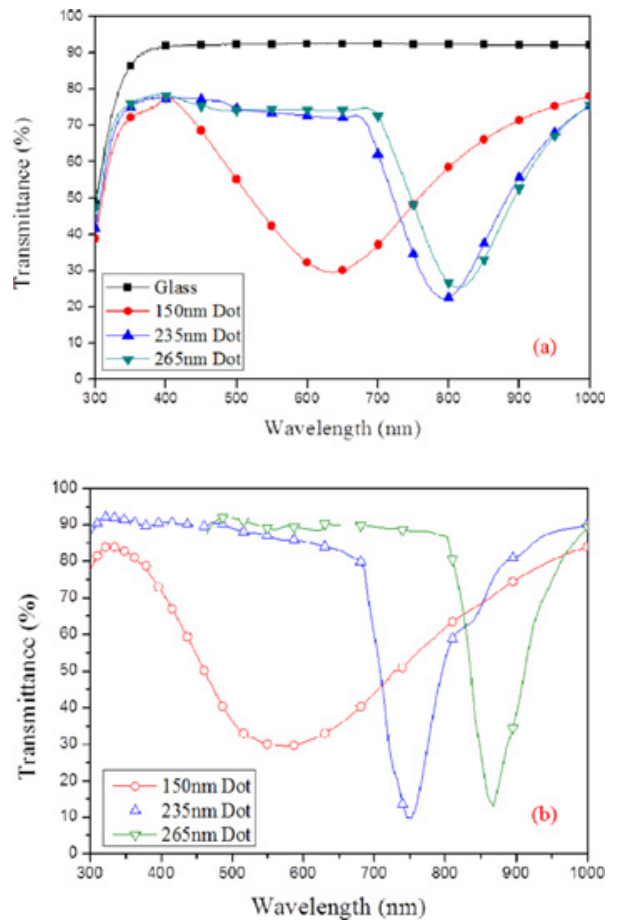

Fig. 5 (a) Transmittance variation with respect to dot diameter $(150 \mathrm{~nm}, 235 \mathrm{~nm}$, and $265 \mathrm{~nm})$ for the patterns shown in Fig. 3; (b) the corresponding simulation results, which appear to be wellmatched to the measurements. 
range of 300-1,000 nm. Corresponding simulations were carried out, and the results are presented in Fig. 5(b) for comparison with Fig. 5(a). Light incidence normal to the sample was picked up on the back side, so that only zero-order transmittance spectra were collected. The corresponding optical transmittance and near electric field distribution simulations for the metal nanodots were performed using the 3D finite difference time domain (FDTD) of Lumerical software. The size of a mesh cell in the simulated structure was $222 \mathrm{~nm}$ (x-, y-, and zdirections). A linearly polarized broadband $(300-1000 \mathrm{~nm})$ plane wave illuminated the metal nanodot structure from the array side at normal incidence. To simulate a periodic array structure, we used periodic boundary conditions along the $\mathrm{x}$ - and $\mathrm{y}$-axes, while perfectly matched layer (PML) boundary conditions were applied in the z-direction. The Lumerical multi-coefficient model was used to fit the empirical dielectric constants of silver, which were taken. ${ }^{25}$

The transmittance spectral profile for the $150 \mathrm{~nm}$ hexagonal silver pattern array, shown in Fig. 5(a), started to decrease at a wavelength of $400 \mathrm{~nm}$, bottomed out at around $640 \mathrm{~nm}$, and then slowly increased at wavelengths up to $1,000 \mathrm{~nm}$. The transmittance dropped by as much as $48 \%$, and its bandwidth was broad up to $600 \mathrm{~nm}$, which is in good agreement with the simulation result shown in Fig. 5(b). On the other hand, narrower bandwidths were obtained for the $235 \mathrm{~nm}$ and $265 \mathrm{~nm}$ silver pattern arrays, approximately $332 \mathrm{~nm}$ and $319 \mathrm{~nm}$ at the center drop-peak wavelengths of $794 \mathrm{~nm}$ and $814 \mathrm{~nm}$, respectively. The transmittance drops were close to $50 \%$, slightly larger than that of the $150 \mathrm{~nm}$ pattern.

The transmittance drop divided by the bandwidth wavelength (temporarily referred to as the localized absorption in this publication) was used to quantify the plasmonic sensing accuracy or emission efficiency at selected illuminative wavelengths. Its respective values for the $150 \mathrm{~nm}, 235 \mathrm{~nm}$, and $265 \mathrm{~nm}$ silver pattern arrays were $0.08,0.15$, and 0.16. Accordingly, the wavelength position at the transmittance drop-peak red-shifted from $637 \mathrm{~nm}$ to $814 \mathrm{~nm}$ with increasing silver dot size, obviously due to the diffraction scattering occurring at the half-pitch wavelength of incidence light. Similar trends were identified in the corresponding simulation results, especially from the perspective of localized absorption and the red-shifting absorption point.

As shown in Fig. 5(b), the simulated rate of transmittance drop decreased from 35 to 26 to $18 \%$ as the dot size increased from 150 to $235 \mathrm{~nm}$ to $265 \mathrm{~nm}$. Thus, the half-width of the band wavelength decreased with increasing dot size, indicating enhanced plasmonic resonance performance. The drop-peak transmittance was $28.8 \%$ for
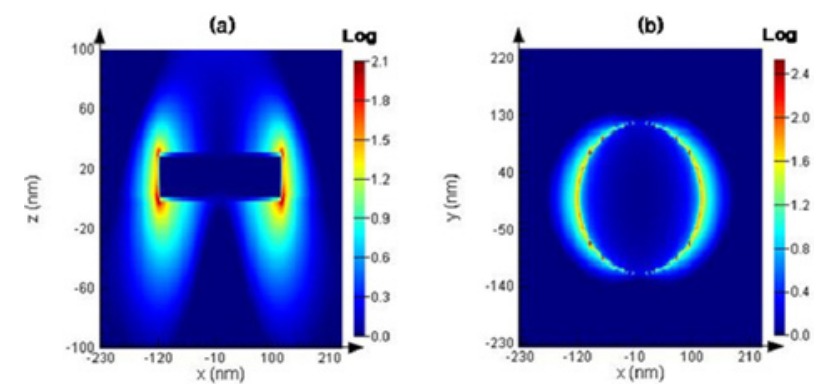

Fig. 6 Simulated electric field distribution and localization results for the $235 \mathrm{~nm}$ metal dot array under resonance conditions: (a) crosssectional and (b) topside views
$150 \mathrm{~nm}, 18.8 \%$ for $235 \mathrm{~nm}$, and $13.7 \%$ for $265 \mathrm{~nm}$, whereas the corresponding wavelength at the transmittance drop-peak red-shifted from 586 to 868 to $914 \mathrm{~nm}$ with increasing dot size. In addition, the socalled localized absorption similarly increased from 0.11 to 0.16 to 0.69 ; this trend is qualitatively in good agreement with our experimental measurements, as well as with others cited above. Quantitatively, however, the results are not well matched. The experimental transmittance at drop-peak wavelength was almost twice as high ( $25.3 \%$ for the $265 \mathrm{~nm}$ dots) as the corresponding simulated value, since resonance absorption was not sufficiently realized, probably in the fabricated silver nanodot array windows, as the simulation reflected. This would also explain the higher localized absorption derived from the simulation data, especially for $265 \mathrm{~nm}$ dot array. Above all, the profile dimensions of the fabricated structure deviated slightly from the master pattern design at each step of the process, as was discussed earlier in connection with Fig. 3. This should critically affect its optical properties with numerical noise errors.

Fig 6 shows the simulated field distribution and localization around a single metal dot with a diameter of $235 \mathrm{~nm}$ under resonance conditions. As the cross-sectional [Fig. 6(a)] and topside [Fig. 6(b)] views indicate, the field was most enhanced at the corners of the structure and along the sidewalls.

\section{Conclusion}

Metal nanodot arrays were fabricated on a glass substrate via an advanced lift-off technology, utilizing a lift-off resist and a Si-based etch mask resist. Hence the Si-based etch mask enabled the lift-off process window to be extended in terms of increased etching dimensional tolerances, providing a more reliable and reproducible fabrication strategy than conventional lift-off. It should be noted that the Si-based resist fills the imprinted pattern and planarizes, in addition to functioning quite effectively as an etch mask. It also contributes to the formation of tapered sidewalls upon etch-down of lift-off of the resist layer. Hence, metal deposition on the sidewalls of the lift-off layer below can be naturally prevented, unless extremely isotropic metal sputtering conditions are intentionally employed. Without this, optimization of the lift-off process conditions becomes more challenging, or produces only a limited range of metal pattern thicknesses, and rabbit ear structures are likely to be created. In the present work, rounded silver dots were successfully fabricated without creating rabbit ears, indicating the possibility of constructing metal nanostructure arrays with high-aspect-ratio thicknesses for the three given master pattern sizes.

To characterize the optical properties of the silver dot arrays, spectroscopic transmittances were measured and compared with simulation results. Analysis of the measurement data essentially reflected homogeneously averaged optical properties on an incident light spot with millimeter-scale area, which could be used to characterize their atmospheric uniformity and repeatability in samples fabricated under identical process conditions. In optoelectronic or sensing applications, homogenized properties on an illumination spot could be somewhat more meaningful for verifying device performance. Hence, the objective of the present work was to indirectly assess the 
dimensional integrity of fabricated silver pattern arrays via spectroscopic optical measurements on the millimeter scale, as well as SEM analysis on virtual nanoscale.

The experimental measurements were qualitatively in fairly good agreement with the simulation data. A slight deviation was attributed to dimensional variations caused by the reverse-tapered profile of the under-etched structure masked by the Si-based resist. Nevertheless, the present approach could be reliably applied to fabricate metal nanodot arrays for a wide range of plasmonic and photonic devices.

\section{ACKNOWLEDGEMENT}

This research was supported by a grant (2011-0028585) from the Basic Science Research Program and a grant (2011-0031563) from the Global Frontier R\&D Program, funded by the National Research Foundation of Korea (NRF) under the Ministry of Education, Science, and Technology of Korea.

\section{REFERENCES}

1. Kang, M. -G., Xu, T., Park, H. J., Luo, X., and Guo, L. J., "Efficiency Enhancement of Organic Solar Cells Using Transparent Plasmonic Ag Nanowire Electrodes," Advanced Materials, Vol. 22, No. 39, pp. 4378-4383, 2010.

2. Zhang, X. L., Feng, J., Song, J. F., Li, X. B., and Sun, H. B., "Grating Amplitude Effect on Electroluminescence Enhancement of Corrugated Organic Light-Emitting Devices,” Opt Lett, Vol. 36, No. 19, pp. 3915-3917, 2011.

3. Feng, J., Okamoto, T., and Kawata, S., "Enhancement of Electroluminescence through a Two-Dimensional Corrugated Metal Film by Grating-Induced Surface-Plasmon Cross Coupling," Optics Letters, Vol. 30, No. 17, pp. 2302-2304, 2005.

4. Reboud, V., Kehagias, N., Kehoe, T., Leveque, G., Mavidis, C., and et al., "Nanoimprinted Plasmonic Crystals for Light Extraction Applications," Microelectronic Engineering, Vol. 87, No. 5-8, pp. 1367-1369, 2010.

5. Maria, J., Truong, T. T., Jimin, Y., Lee, T. -W., NUZZO, R. G., and et al., "Optimization of 3D Plasmonic Crystal Structures for Refractive Index Sensing” J. Phys. Chem. C, Vol. 113, No. 24, pp. 10493-10499, 2009.

6. Yao, J., Stewart, M. E., Maria, J., Lee, T. -W., Gray, S. K., and et al., "Seeing Molecules by Eye: Surface Plasmon Resonance Imaging at Visible Wavelengths with High Spatial Resolution and Submonolayer Sensitivity," Angewandte Chemie International Edition, Vol. 47, No. 27, pp. 5013-5017, 2008.

7. Stewart, M. E., Mack, N. H., Malyarchuk, V., Soares, J. A. N. T., Lee, T. W., and et al., "Quantitative Multispectral Biosensing and 1D Imaging using Quasi-3D Plasmonic Crystals," Proc. Natl. Acad. Sci., Vol. 103, No. 46, pp. 17143-17148, 2006.
8. Gupta, R. K., Krishnamoorthy, S., Kusuma, D. Y., Lee, P. S., and Srinivasan, M. P., "Enhancing Charge-Storage Capacity of NonVolatile Memory Devices using Template-Directed Assembly of Gold Nanoparticles," Nanoscale, Vol. 4, No. 7, pp. 2296-2300, 2012.

9. Yun, D. K., Kim, K. D., Jeong, H. Y., Lee, J. H., Jeong, J. H., and Choi, S. Y., "Fabrication of TiO2 Memristive Arrays by Step and Flash Imprint Lithography," J. Nanosci Nanotechnol, Vol. 11, No. 1, pp. 696-700, 2011.

10. Cui, B., Clime, L., Li, K., and Veres, T., "Fabrication of large area nanoprism arrays and their application for surface enhanced Raman spectroscopy," Nanotechnology, Vol. 19, No. 14, pp. 145302, 2008.

11. Singh, M., Srivastava, S., Agarwal, S., Kumar, S., and Vijay, Y. K., "Optical Properties of D.C. Magneto Sputtered Tantalum and Titanium Nanostructure Thin Film Metal Hydrides," Bulletin of Materials Science, Vol. 33, No. 5, pp. 569-573, 2010.

12. Ting, C. J., Chen, C. F., and Hsu, C. J., "Heat-insulation film with gold layer deposited on antireflection subwavelength-structured surface," Optik - International Journal for Light and Electron Optics, Vol. 121, No. 20, pp. 1877-1880, 2010.

13. Kim, E. U., Baeg, K. J., Noh, Y. Y., Kim, D. Y., Lee, T., Park, I., and Jung, G. Y., "Templated Assembly of Metal Nanoparticles in Nanoimprinted Patterns for Metal Nanowire Fabrication," Nanotechnology, Vol. 20, No. 35, Paper No. 355302, 2009.

14. Xia, G., Wang, S., and Jeong, S. J., "A Universal Approach for Template-Directed Assembly of Ultrahigh Density Magnetic Nanodot Arrays," Nanotechnology, Vol. 21, No. 48, Paper No. 485302, 2010.

15. Lim, K. M., Gupta, S., Ropp, C., and Waks, E., "Development of Metal Etch Mask by Single Layer Lift-Off for Silicon Nitride Photonic Crystals," Microelectronic. Eng., Vol. 88, No. 6, pp. 994998, 2011.

16. De Angelis, F., Liberale, C., Coluccio, M. L., Cojoc, G., and Di Fabrizio, E., "Emerging Fabrication Techniques for 3D NanoStructuring in Plasmonics and Single Molecule Studies," Nanoscale, Vol. 3, No. 7, pp. 2689-2696, 2011.

17. Hindmarch, A. T., Parkes, D. E., and Rushforth, A. W., "Fabrication of Metallic Magnetic Nanostructures by Argon Ion Milling using a Reversed-Polarity Planar Magnetron Ion Source," Vacuum, Vol. 86, No. 10, pp. 1600-1604, 2012.

18. Mekaru, H. and Takahashi, M., "Imprinting a Needle Array on a Polycarbonate Substrate" Int. J. Precis. Eng. Manuf., Vol. 10, No. 10, pp.79-83, 2011.

19. Chen, Y., Macintyre, D. S., and Thoms, S., "A Non-Destructive Method for the Removal of Residual Resist in Imprinted Patterns" Microelectronic. Eng., Vol. 67-68, pp. 245-251, 2003.

20. Carlberg, P., Graczyk, M., Sarwe, E. L., Maximov, I., Beck, M., and Montelius, L., "Lift-Off Process for Nanoimprint Lithography," Microelectronic Engineering, Vol. 67-68, pp. 203-207, 2003. 
21. Chen, Y., Peng, K., and Cui, Z., "A Lift-Off Process for High Resolution Patterns using PMMA/LOR Resist Stack," Microelectronic Engineering, Vol. 73-74, pp. 278-281, 2004.

22. Shields, P. A., and Allsopp, D. W. E., "Nanoimprint Lithography Resist Profile Inversion for Lift-Off Applications," Microelectronic Engineering, Vol. 88, No. 9, pp. 3011-3014, 2011.

23. Buyukkose, S., Vratzov, B., and van der Wiel, W. G., "High-Quality Global Hydrogen Silsequioxane Contact Planarization for Nanoimprint Lithography" J. Vac. Sci. Technol. B., Vol. 29, No. 2, pp.1602-1606, 2011.

24. Matsui, S., Igaku, Y., Ishigaki, H., Fujita, J., Ishida, M., and et al., "Room Temperature Replication in Spin on Glass by Nanoimprint Technology," Journal of Vacuum Science \&amp; Technology B, Vol. 19, No. 6, pp. 2801-2805, 2001.

25. E. D. Palik, "Handbook of Optical Constants of Solids," Academic, 1985. 